\title{
Keyword Search over RDF Using Document-Centric Information Retrieval Systems
}

\author{
Giorgos Kadilierakis ${ }^{1,2}$, Pavlos Fafalios ${ }^{1(\bowtie)}$ (D), Panagiotis Papadakos ${ }^{1,2}$ (D), \\ and Yannis Tzitzikas ${ }^{1,2}$ (D) \\ 1 Information Systems Laboratory, FORTH-ICS, Heraklion, Greece \\ kadilier@csd.uoc.gr, \{fafalios,papadako,tzitzik\}@ics.forth.gr \\ ${ }^{2}$ Computer Science Department, University of Crete, Heraklion, Greece
}

\begin{abstract}
For ordinary users, the task of accessing knowledge graphs through structured query languages like SPARQL is rather demanding. As a result, various approaches exploit the simpler and widely used keyword-based search paradigm, either by translating keyword queries to structured queries, or by adopting classical information retrieval (IR) techniques. In this paper, we study and adapt Elasticsearch, an out-ofthe-box document-centric IR system, for supporting keyword search over RDF datasets. Contrary to other works that mainly retrieve entities, we opt for retrieving triples, due to their expressiveness and informativeness. We specify the set of functional requirements and study the emerging questions related to the selection and weighting of the triple data to index, and the structuring and ranking of the retrieved results. Finally, we perform an extensive evaluation of the different factors that affect the IR performance for four different query types. The reported results are promising and offer useful insights on how different Elasticsearch configurations affect the retrieval effectiveness and efficiency.
\end{abstract}

\section{Introduction}

The Web of Data currently contains thousands of RDF datasets available online that includes cross-domain KBs like DBpedia and Wikidata, domain specific repositories like DrugBank and MarineTLO, as well as Markup data through schema.org (see [17] for a recent survey). These datasets are queried through structured query languages (SPARQL), however this is quite complex for ordinary users. Ordinary users are acquainted with keyword search due to the widely used web search engines. Faceted search system is another popular paradigm for interactive query formulation, however even such systems (see [24] for a survey) need a keyword search engine as an entry point to the information space. We conclude that an effective method for keyword search over RDF is indispensable. 
At the same time we observe a widespread use of out-of-the-box IR systems, like Elasticsearch, in different contexts. To this end in this paper we investigate how such existing document-centric Information Retrieval Systems (IRSs), can be used for enabling keyword search over arbitrary RDF datasets, and how they perform compared to dedicated keyword search systems for RDF. Towards this aim, we study the following relevant questions: (a) how to index an RDF dataset, (b) what data we should rank and how, and (c) how the search results should be presented. In this work, we study and propose various methods for tackling the above questions over the popular IR system Elasticsearch, and report extensive evaluation results in terms of their effectiveness and efficiency.

The source code of our implementation is available on GitHub as an indexing service $^{1}$ and a search $\mathrm{API}^{2}$. We also provide a demo named Elas $4 \mathrm{RDF}^{3}$ on top of these services over the DBpedia dataset, where the services are configured based on the most effective options reported in this work.

The rest of the paper is organized as follows: Sect. 2 describes the related background, requirements and challenges, Sect. 3 discusses the related work, and Sect. 4 details our adaptation of Elasticsearch for RDF. Finally, Sect. 5 discusses the evaluation results, while Sect. 6 concludes the paper and identifies issues for further research.

\section{Problem Statement and Requirements}

Section 2.1 describes the background and the main objective, Sect. 2.2 discusses the requirements, and Sect. 2.3 identifies the rising questions and challenges.

\subsection{Background and Objective}

We first define the notions of $R D F$ triple and $R D F$ dataset. Consider an infinite set of URI references $\mathcal{U}$, an infinite set of blank nodes $\mathcal{B}$ (anonymous resources), and an infinite set of literals $\mathcal{L}$. A triple $\langle s, p, o\rangle \in(\mathcal{U} \cup \mathcal{B}) \times \mathcal{U} \times(\mathcal{U} \cup \mathcal{L} \cup \mathcal{B})$ is called an RDF triple, where $s$ is the subject, $p$ the predicate, and $o$ the object of the triple. An RDF dataset (or RDF graph) is a finite set of RDF triples. These triples usually describe information for a set of entities $E$ (subject or object URIs), like persons, locations, etc. Figure 1 depicts an example of a small RDF graph describing three albums of The Beatles band. It contains 16 triples, involving 4 entity URIs (black nodes), 2 class URIs (white nodes), and 8 literals (gray nodes). Among the 8 literals, 7 are strings (free text) and 1 is a number.

Our objective is to allow a user submit a free-text query $q$ and get back the most relevant data, for a given set of RDF triples $T$.

\footnotetext{
${ }^{1}$ https://github.com/SemanticAccessAndRetrieval/Elas4RDF-index.

${ }^{2}$ https://github.com/SemanticAccessAndRetrieval/Elas4RDF-search.

${ }^{3}$ https://demos.isl.ics.forth.gr/elas4rdf.
} 


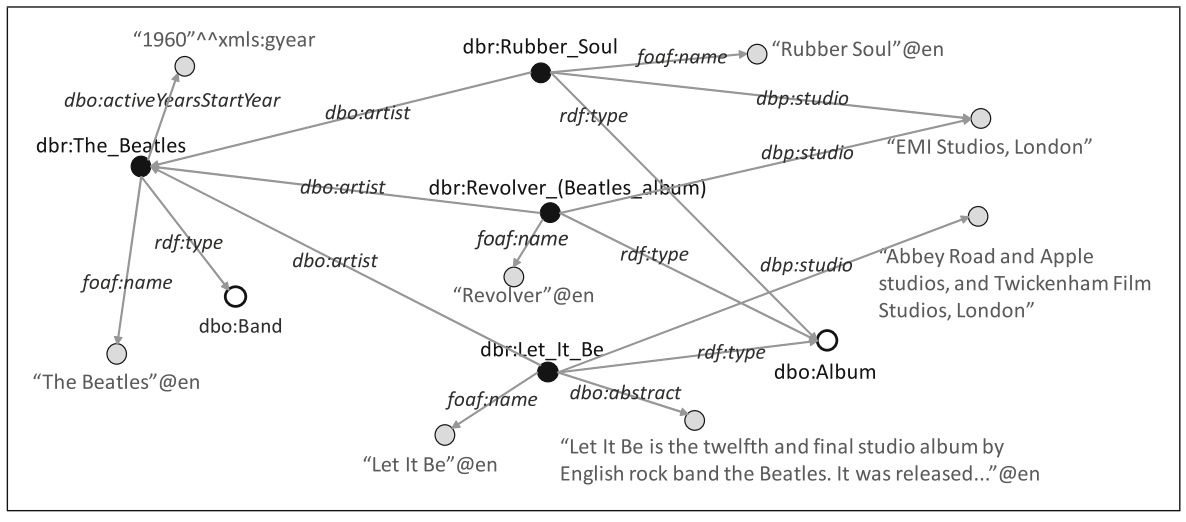

Fig. 1. An example of a small RDF graph.

\subsection{Requirements}

We consider the following three functional requirements:

- Unrestricted RDF datasets. A valid RDF dataset contains any set of valid RDF triples. We do not presuppose knowledge of the ontology/schema for describing the underlying data. Thus, triples describing the data schema may not exist. In addition, the dataset might not contain human-friendly URIs.

- Unrestricted keyword-based/free-text queries. The only input is a free-text query describing any type of information need (e.g., retrieving an entity, attributes of an entity, etc.). We do not consider query operators like AND/OR, wildcards, the ability to search in specific indexed fields, phrasal queries, or any other input specified at query-time.

- Exploitation of an existing IR system. We do not aim at building a new IR system. Instead we want to use an existing widely-used system, exploit its functionalities, and tune it for retrieving RDF data. Whenever possible, we should use its default settings. Any configuration should be made only if this is required by the nature of the RDF data, but without considering any information about the topic or domain of the indexed dataset.

\subsection{Challenges}

We can identify four basic challenges of keyword search over RDF data:

Challenge 1: Deciding on the Retrieval Unit. Contrary to the classic IR task where the retrieval unit is an unstructured or semi-structured textual document, an RDF dataset contains highly-structured data in the form of RDF triples, where each triple consists of three elements: subject, predicate and object. There are three main options to consider regarding the retrieval unit:

(i) An entity corresponding to a single URI. An RDF dataset usually describes information for a set of resources (e.g., persons or locations). Such a resource can be found either in the subject and/or the object of the triple, 
and satisfies entity search information needs, related to the retrieval of one or more entities, like the query "The Beatles albums".

(ii) A triple (subject-predicate-object). It provides more information than single URIs, satisfying information needs related to attribute search. In such tasks we want to find an attribute of an entity (e.g., "Beatles formation year"), or general information of an entity as captured by string literals. The triple can also help verify the correctness of a result, e.g., (dbr:The_Beatles, dbo:artist, dbr:Let_It_be) for the query "Artist of Let It Be", instead of returning the URI of an entity like dbr: The_Beatles.

(iii) A subgraph (of size $\boldsymbol{l}$ triples). It describes more complex information than a single triple. Consider the query "Beatles studios". The answer consists of the two literals ("EMI Studios...", "Abbey Road..."), connected to the Beatles' albums Revolver and Rubber Soul through the property dbp:studio, which in turn are connected to The Beatles entity through the property dbo:artist. Thus, a correct candidate answer consists of a path or subgraph of two triples: <dbr:The_Beatles, dbo:artist, dbr:Rubber_Soul> and <dbr:Rubber_Soul, dbp:studio, "EMI Studios, London">.

Challenge 2: Selecting the Data to Index. An RDF dataset contains elements of different types: i) resource identifiers (URIs/URLs), ii) string literals, iii) numerical and boolean literals, iv) date literals, and v) unnamed elements (blank nodes) that are used for connecting other elements. Types ii-iv are all literals, so there is no need for any special preprocessing, while blank nodes (type v) can be ignored. With respect to type i, the last part of a URI usually reveals the name of the corresponding entity or resource, and is rather useful after some pre-processing (e.g., replacing underscores with space). The domain of the URI usually reveals the knowledge base it belongs to, e.g., DBpedia, and its middle part can reveal the type of the resource (e.g., class, property, etc.), which can be useful for more experienced users. If the retrieval unit is an entity, one can index (parts of) its URI as well as all its outgoing properties that provide characteristics and more information about the entity. If the retrieval unit is a triple, one can just index all of its parts (subject, predicate, object), or choose to index additional data about the subject and object of the triple, e.g., literal properties like the rdfs: label. Finally, if we consider a subgraph as the retrieval unit, then the data to index depends on whether the subgraph has a constant size, independently of the query, or its size is selected dynamically. For the former, one storage-inefficient option is to index all possible subgraphs of size $l$. Thus, a more flexible approach is to index single triples and select the $l$ triples that form a subgraph during the retrieval process.

Challenge 3: Weighting the Indexed Fields. Deciding on the importance of each indexed field may be another thing to consider. By assigning weights, important fields can affect more the final ranking. For example, we may assign higher weights to URI's containing certain properties (e.g., label, comment, etc.), or to 
literals over URIs. By allowing the adjustment of weights of the various fields at query time, we can fine-tune the IRS's query evaluator module at run-time, offering better results for easily identifiable query types (e.g., Q\&A queries).

Challenge 4: Structuring the Results. The final challenge is to decide on how to structure and show the results page. One option is to follow a classical IR approach and show a top-K ranked list of individual results (i.e., entities, triples or subgraphs), and its metadata (e.g., relevance score) through a faceted search UI. Another option is to show a top-K graph which depicts how the individual results (entities, triples, or subgraphs), are connected to each other.

We study all these challenges as parameters of Elasticsearch (see Sect.4).

\section{Related Work}

Keyword search over RDF data can be supported either by translating keyword queries to structured (SPARQL) queries (like in $[8,15,22,23]$ ), or by building or adapting a dedicated IRS using classical IR methods for indexing and retrieval.

Since our work falls under the second direction, below we report related works and showcase the difference of our approach. Such systems construct the required indexing structures either from scratch or by employing existing IR engines (e.g., Lucene and Solr), and adapt the notion of a virtual document for the structured RDF data. Usually, they rank the results (entities or subgraphs) according to commonly used IR ranking functions. One of the first such systems was Falcon [2], where each document corresponds to the textual description of the maximum subset of connected RDF triples, while the ranking of the documents is done by mapping keyword terms to documents through cosine similarity and the popularity of each document. In the entity search track of SemSearch10 workshop ${ }^{4}$, a number of related systems were presented and evaluated $[4,5,16]$. Most of those systems are based on variations of the TD-IDF weighting adapted for RDF data, and return a ranked list of entities (i.e., URIs). An approach that uses inverted lists over terms that appear as predicates or objects of triples is described in [3], where the keyword query is translated to a logical expression that returns the ids of the matching entities. Another direction in the bibliography is to return ranked subgraphs instead of relevant entity URIs. For example, in [18] documents represent a literal or a resource, and external knowledge is used to explore relations between the keywords and the dataset components, while the returned subgraphs are ranked using a TF-based function. In [7] the returned subgraphs are computed using statistical language models based on the likelihood estimation of generating the query from each subgraph.

In current state-of-the-art approaches though, RDF data are ranked based on extensions of the BM25 model. For example, BM25F [1,19], takes into account the various fields of a virtual document and computes the normalized termfrequency using the field's length instead of the document's. Further, the recent work described in [6] introduces the TSA + VDP keyword search system, where

\footnotetext{
${ }^{4}$ http://km.aifb.kit.edu/ws/semsearch10/.
} 
initially, the system builds offline an index of documents over a set of subgraphs via a breadth-first search method, while at query-time, it returns a ranked list of these documents based on a BM25 model.

Regarding the retrieval unit, we have seen that most works return either URIs or subgraphs. However, the concept of triple ranking has also emerged in works that do not directly target the task of keyword search over RDF data. For example, the TripleRank algorithm presented in [9] ranks authorities in the Semantic Web, in the same manner as PageRank for the WWW. In [20], the authors propose a learning to rank framework with relation-independent features that aims at developing ranking models that measure triple significance. For a given relation type as input (e.g., profession) the computed score of each triple measures how well the triple captures the relevance of the statement that it expresses, compared to other triples from the same relation.

With respect to works that make use of Elasticsearch, LOTUS $[11,12]$ is a text-based entry point to the Linked Data cloud. It makes use of Elasticsearch for supporting keyword search, offering various approaches for matching and ranking the relevant information. Its focus is on scalability and does not study how the different matching and ranking methods affect the retrieval performance. Elasticsearch has been also used for indexing and querying Linked Bibliographic Data in JSON-LD format [14], while the ElasticSearch RDF River Plugin ${ }^{5}$ uses it as a way to index URIs from various endpoints and enrich the indexed documents with RDF data. ${ }^{6}$

Positioning. In our work, we make use of Elasticsearch for supporting schema-agnostic keyword search over a set of RDF triples, in order to return a ranked list of triples. We also provide ways of constructing a ranked list of entities over this list of triples. Complementary to the approach followed by LOTUS $[11,12]$, which focuses on the scalability and efficiency of query evaluation using Elasticsearch, we study in detail how the various configuration options affect the retrieval accuracy. We aim at gaining a better understanding on how Elasticsearch performs over RDF, so that anyone can use it out-of-thebox over any RDF dataset. Our experimental evaluation (Sect.5) showed that, a proper (schema-agnostic) configuration in Elasticsearch provides a retrieval accuracy similar to that of dataset-specific approaches built from scratch for the task per se. To our knowledge, our work is the first that studies how the different indexing and retrieval options in Elasticsearch affect the retrieval accuracy.

\section{Adapting a Document-Centric IRS for RDF}

Here, we describe the selected IRS (Sect.4.1) and provide an overview of our approach (Sect. 4.2). Then, we detail the various options we experimented with, regarding indexing (Sect.4.3), retrieval (Sect.4.4) and ranking (Sect.4.5).

\footnotetext{
${ }^{5}$ https://github.com/eea/eea.elasticsearch.river.rdf\#main-features.

${ }^{6}$ https://www.opensemanticsearch.org/connector/rdf.
} 


\subsection{Considered IRS: Elasticsearch}

Elasticsearch is a highly-scalable, open-source, full text search engine that allows to store and search big volumes of data. It is based on Apache Lucene and offers a distributed architecture of inverted indexes.

Basic Concepts. All data in Elasticsearch are stored in indices containing different types of documents (units of search and index) that Elasticsearch can store, update and search. Each document is a JSON object stored with a unique ID that contains a set of fields. Each field is a key-value pair of various datatypes (e.g., strings, JSON objects, etc.), organized by a mapping type for each index. In our work, we create different mappings depending on the approach we follow. For each field, we need to specify a type (e.g., text) and an analyzer, and also define the used tokenizer, stemmer and stopword-list. Each index can be split into multiple shards, and each shard can be replicated using replicas. A node contains multiple shards/replicas and if the number of nodes is greater than one, Elasticsearch balances the load equally. Finally, a single cluster may contain one or more nodes that run in parallel and serve multiple requests.

Query Domain Specific Language (DSL). Elasticsearch has a powerful Query DSL which supports advanced search features on top of Lucene's query syntax. There are two main types of query clauses: (a) filter-context which answers whether a query matches a document (exact-match), and (b) querycontext which answers how well does a document matches a query using a relevance score (best-match). Since we are interested in free-text search that provides a ranked-list of results, we will solely be using query-context clauses. Queries can be further categorised in match queries and multi-match queries. A match query is executed over a single field, while a multi-match query allows searching upon multiple fields. Depending on the way it is executed internally, a multi-match query is categorized in: (i) best-fields, (ii) most-fields and (iii) cross-fields. Types (i) and (ii) follow a field-centric approach, evaluating all query keywords on each field before combining scores from each field. Type (i) assigns as document score the score of the best-matched field, while for type (ii), the final score is the average score of all field scores. Field-centric approaches appear to be problematic in cases where the query terms are scattered across multiple fields (e.g., across the triple's subject, predicate and object). A term-centric approach addresses this issue by searching a query term-by-term on each field. This is implemented in type (iii) where cross-fields searches each term across fields, favoring queries whose answer is scattered across multiple fields.

\subsection{Overview of the Approach}

In this section we describe how we cope with the challenges discussed in Sect. 2.3, and provide an overview of our approach and implementation.

With respect to Challenge 1 (deciding on the retrieval unit), we opt for high flexibility and thus consider triple as the retrieval unit. A triple is more informative than an entity, provides a means to verify the correctness of a piece 
of information, since it is closer to Q\&A, and offers flexibility on how to structure and present the final results (Challenge 4). For example, one can use various aggregation methods over a ranked list of retrieved triples, for providing a ranked list of entities for entity search or showing graphs of connected entities. Moreover in $R D F$, a triple can be viewed as the simplest representation of a fact. This property is one of the major reasons we chose triple as our virtual document.

Regarding Challenge 2 (selecting the data to index), we experiment and evaluate different approaches on what data to consider for each virtual document. Our baseline approach, considers only data from the triple itself (i.e., text extracted from the subject, object and predicate). This simple approach, may appear problematic in a dataset where URI's are IDs, and thus not descriptive of the underlying resource. As a result, we also extend the baseline approach to exploit information in the neighborhood of the triple's elements. For example, we consider important outgoing properties such as rdfs:label and rdfs:comment, and evaluate how various extensions affect the results quality and the index size.

With respect to Challenge 3 (weighting the indexed fields), we do not apply any predefined weights in the indexed fields, but instead, adjust the weights of the various fields at query time. In this way, the IRS's query module evaluator can be fine-tuned at run-time for specific query types (e.g., Q\&A queries).

Finally, for Challenge 4 (results structuring), we opt for a ranked-list of results since this is the way that traditionally IRS present the results to the user. On top of the ranked-list of triples, we propose a method for mapping the retrieved triples into a ranked list of entities, based on the appearance of URIs either in the subject or the object. Then, the entities are ranked based on a weighted gain factor of the ranking order of the triples in which they appear, similar to the discounted cumulative gain used in the $\mathrm{nDCG}$ metric [13]. The evaluation of different visualization methods (e.g., list of resources, top-K graphs, etc.) and the corresponding user experience go beyond the scope of this paper.

Below, we provide details of the different approaches we experimented with for indexing, retrieval and ranking of RDF triples in Elasticsearch.

\subsection{Indexing}

We try variations of two different indexing approaches, the baseline index that considers only the triple itself, and the extended index that extends the baseline index with additional descriptive information about the triple components.

Baseline Index. This index uses only information that exists in the triple's three components (subject, predicate, object). In case the value of one of the components is a URI, the URI is tokenized into keywords, based on a special tokenizer that extracts the last part of the URI (i.e., the text after the last '/' or ' $\#$ ') that usually describes the underlying resource, and its namespace parts.

Extended Index. The extended index, includes additional information when one of the triple components is a resource (URI). This is particularly useful when the last part of the URIs are not descriptive of the corresponding resources, and thus not useful for querying. We experiment with three different variations that 
include the value(s) of: i) the rdfs:label property, that usually contains the name of the corresponding resource, ii) the rdf s : comment property, which is very descriptive of the underlying resource, and provides much more information than the rdfs:label, and iii) all the outgoing properties of the resource. The first two approaches are useful when we are aware of the schema(s) used to describe the data. The latter one, includes all the information that describes the resource. However it can highly increase the size of the index and introduce noise.

\subsection{Retrieval}

We experimented with various query types, weighting methods and similarity models offered by Elasticsearch.

Query Types. Since our indexes contain different sets of fields, we can use multiple types of Elasticsearch queries. We study the following two approaches: (i) single-field: a single field is created containing the index data, e.g., a superfield containing all keywords describing the subject, the predicate and the object, and (ii) multi-field: multiple fields are created, each one containing a specific piece of information, e.g., one field for the subject keywords, one for the predicate keywords and one for the object keywords.

Weighting. Another factor for improving relevance at query retrieval time is applying weights on the various fields. Boosting fields only makes sense upon multi-field queries, for specifying the importance of a field over another. For example, we may define that the field containing the object keywords is twice more important than the fields containing the subject and predicate keywords. We experimented with different weighting approaches, by weighting more either: i) only the subject keywords; ii) only the object keywords; iii) both the subject and the object keywords.

Similarity Models (and Parameterization). A similarity model defines how matching documents are scored. In Elasticsearch the default model is Okapi BM25, which is a TF/IDF based similarity measure. BM25 has an upper limit in boosting terms with a high TF, meaning that it follows a nonlinear term frequency saturation. Parameter $k 1$ can control how quickly this saturation will happen based on the TF value. The default value is 1.2 and higher values result in slower saturation. In our case, since the text in our fields is generally short, $k 1$ will probably perform better towards lower values. The other tuning option of BM25 is the field-length normalization, that can be controlled with parameter $b$ which has a default value of 0.75 . Shorter fields gain more weight than longer fields by increasing $b$, and this can be used to boost a short descriptive resource over a long literal inside an object field.

Another available similarity module in Elasticsearch is DFR, a probabilistic model that measures the divergence from randomness. Parameters include a basic randomness model definition, using inverse term frequency, and a twolevel normalization. Language models supported by Elasticsearch include the $L M$-Dirichlet similarity, a bayesian smoothing that accepts the $\mu$ parameter, 
and the LM-Jelinek Mercer similarity, which can be parameterized with $\lambda$. We experimented with all the above-mentioned similarity models.

\subsection{Grouping and Final Ranking}

At this point we have performed a keyword query and have retrieved a ranked list of triples (1st-level results). Now, we need to decide on how we will present the results to the user. One approach is to group the retrieved triples based on entities (i.e. subject and object URIs), and return a ranked list of entities (2ndlevel results) to the user, where each entity is associated with a ranked list of triples. Such an approach offers flexibility on how to display the results to the user, and allows to evaluate the different configurations we experimented with using as ground truth existing datasets for entity search [10] (more below).

For ranking the derived entities, we exploit the ranking order of the triples based on a weighted factor. Thereby, the gain that each entity accumulates works in a logarithmic reduction manner, as in the widely used Discounted Cumulative Gain (DCG) metric [13]. Specifically, each entity collects the discounted gain of each triple based on the ranking position that it appeared on the 1st-level results ranking. The final score of an entity $e$ for a keyword-query $q$ is given by the formula:

$$
\operatorname{score}(e, q)=\sum_{t_{i}}^{t_{n}} \frac{2^{\left(\text {n_score }_{i}\right)}-1}{\log _{2}(i+1)}
$$

where $t$ is the ranked list of triples that the entity $e$ appears in, and $n_{-} s c o r e_{i}$ is the normalized score of triple $i$ in that list for the query $q$. Since Elasticsearch deliberately scores documents with any number $>0$, we use minmax normalization for the results in list $t$.

Table 1. Query categories in the 'DBpedia Entity' test collection for entity search.

\begin{tabular}{l|l|l|c}
\hline Category & Description & Example & \# queries \\
\hline SemSearch_ES & Named entity queries & "brooklyn bridge" & 113 \\
\hline INEX-LD & IR-style keyword queries & "electronic music genres" & 99 \\
\hline QALD2 & Natural language questions & "Who is the mayor of Berlin?" & 115 \\
\hline ListSearch & Entity-list queries & "Professional sports teams in New York" & 140 \\
\hline
\end{tabular}

\section{Evaluation}

In Sect.5.1 we describe the setup and the dataset of the evaluation, while Sect. 5.2 and Sect. 5.3 report retrieval effectiveness, and space and time efficiency, respectively. Finally, Sect. 5.4 summarizes the key findings. 


\subsection{Test Collection and Setup}

For our experiments we used the DBpedia-Entity test collection for entity search [10], which is based on a DBpedia dump of 2015-10. The collection contains a set of heterogeneous keyword queries along with relevance judgments obtained using crowdsourcing. There are four categories of queries: i) named-entity queries, ii) IR-style keyword queries, iii) natural language questions, and iv) entity-list queries. Table 1 provides an example and the total number of queries for each query category. In total, over $49 \mathrm{~K}$ query-entity pairs are labeled using a threepoint scale (0: irrelevant, 1: relevant, and 2: highly relevant).

After following the instructions in [10] for building the RDF dataset and removing duplicates, we end up with a collection of approximately 400M triples. In addition to this full-collection, we also generated a subset of 15 million triples that forms our mini-collection by extracting all judged entity-based triples $(\approx 6 \mathrm{M})$ and randomly adding an extra of $9 \mathrm{M}$ unjudged triples. The mini-collection allows us to run a large number of experiments and study how the different factors discussed in the previous section affect the quality of the retrieved results.

We deployed Elasticsearch 6.4 as a single node with max heap size set at 32 GB and 6 physical cores running on Debian 9.6. Using Python's multiprocessing pool we initiate 12 indexing instances with a bulk-size of 3,500 documents each. These numbers were assigned empirically based on the collection and our hardware. The number of shards is also assigned empirically and it alters between the baseline and the extended index. For the baseline we select 2 shards while depending on the extended approach we alter between 3 and 4 shards.

\subsection{Quality of Retrieval}

Our objective is to measure how the following parameters affect the quality of search results: i) the various decisions regarding the indexed triple data, ii) the used Elasticsearch query type, iii) the weighting of the fields, iv) the additional indexed data for each triple, and iv) the available similarity models in Elasticsearch. We first study the effect of all these parameters using the minicollection and then evaluate the best performing methods on the full-collection. For measuring the quality, we make use of the evaluation metric nDCG in positions 100 and 10, as in [10].

Examining Field Separation and Query Type (Baseline-Index). We start by examining how each part of the triple (subject, predicate, object) and the different query types (single field, multi-field) affect the quality of the retrieved results using the baseline index, i.e., without considering additional information about the triple subject, predicate or object. Specifically, we examine the following cases: i) baseline (s): only the keywords of the subject are indexed, ii) baseline $(p)$ : only the keywords of the predicate are indexed, iii) baseline $(o)$ : only the keywords of the object are indexed, iv) baseline (spo): the keywords of all triple's elements are indexed as a single field, v) baseline $(s)(p)(o)$ : the keywords of all triple's elements are indexed as different fields. Single-field queries are executed using the match query retrieval method while multi-fields using multi-match query and cross-fields. 
Table 2 shows the results. As expected, better results are obtained when all triple elements are indexed. The use of a super-field (spo) seems to perform slightly better in average than using distinct fields, mostly for the query types of SemSearch \& INEX-LD. However, the ListSearch type has the best performance when using only the object field, while the Q\&A type when the three fields are distinguished. Recall that, as described in Sect.4.1, the cross-fields query evaluation type of Elasticsearch favors queries whose answer is scattered across multiple fields. This means that in the Q\&A query type, the best results come from more than one fields. With respect to the distinct triple elements, we see that considering only the object provides the best results, outperforming the case where we consider only the subject, by more than $14 \%$. This means that the answer usually exists in the object part of the triple. It is interesting also that considering only the object provides better results than considering all the triple elements for the ListSearch queries. Finally, considering only the predicate provides a very poor performance, being relevant mostly to the Q\&A and ListSearch query types.

Examining Field Weighting (Baseline-Index). Multiple-field queries allow specifying custom weights, enabling us to boost the importance of specific fields. We examine the following cases: i) baseline $(s)^{2}(p)(o)$ : doubling the weight of subject, ii) baseline $(s)(p)(o)^{2}$ : doubling the weight of object, and iii) baseline $(s)^{2}(p)(o)^{2}$ : doubling the weight of both subject and object. Table 3 shows the results. We see that doubling the weight of the object keywords provides the best results on average, slightly outperforming both baseline (spo) and baseline $(s)(p)(o) @ 100$ (cf. Table 2). On the contrary, we notice that doubling the importance of the subject keywords drops the performance by around $10 \%$. Thus, we can conclude that, for this collection, object keywords are more useful for keyword searching than subject keywords.

Table 2. nDCG@100 (@10) for different field separation and query type approaches.

\begin{tabular}{l|l|l|l|l|l}
\hline Method & SemSearch_ES & INEX-LD & QALD2 & ListSearch & AVG \\
\hline Baseline (s) & $0.48(0.46)$ & $0.28(0.26)$ & $0.30(0.20)$ & $0.30(\mathbf{0 . 3 0})$ & $0.340(0.270)$ \\
\hline Baseline (p) & $0.02(0.00)$ & $0.04(0.01)$ & $0.06(0.03)$ & $0.07(0.03)$ & $0.04(0.01)$ \\
\hline Baseline (o) & $0.63(0.50)$ & $0.43(0.30)$ & $0.42(0.26)$ & $\mathbf{0 . 4 7}(0.26)$ & $0.485(0.330)$ \\
\hline Baseline (spo) & $\mathbf{0 . 7 0}(\mathbf{0 . 6 1})$ & $\mathbf{0 . 4 5 ( 0 . 3 3 )}$ & $0.43(0.30)$ & $0.44(0.26)$ & $\mathbf{0 . 5 0 5}(\mathbf{0 . 3 7 2})$ \\
\hline Baseline (s)(p)(o) & $0.65(0.55)$ & $0.44(0.32)$ & $\mathbf{0 . 4 5 ( \mathbf { 0 . 3 1 } )}$ & $0.46(0.28)$ & $0.500(0.358)$ \\
\hline
\end{tabular}

Extending the Index. We now study the case where we extend the index with additional information about the triple's elements. We consider the best performing weighting method, i.e. $(\mathrm{s})(\mathrm{p})(\mathrm{o})^{2}$, and examine the cases described in Sect.4.3: i) extended-label, that includes the rdfs:label property value of the subject and object URIs as two different fields, ii) extended-comment, that includes the rdfs:comment property value of the subject and object URIs as two different fields, and iii) extended-outgoing, that includes the values of all the 
Table 3. nDCG@100 (@10) for different field weighting approaches.

\begin{tabular}{l|l|l|l|l|l}
\hline Method & SemSearch_ES & INEX-LD & QALD2 & ListSearch & AVG \\
\hline Baseline $(\mathrm{s})^{2}(\mathrm{p})(\mathrm{o})$ & $0.54(0.50)$ & $0.36(0.31)$ & $0.36(0.28)$ & $0.36(0.23)$ & $0.405(0.330)$ \\
\hline Baseline $(\mathrm{s})(\mathrm{p})(\mathrm{o})^{2}$ & $\mathbf{0 . 6 7}(\mathbf{0 . 5 5})$ & $\mathbf{0 . 4 5}(0.31)$ & $\mathbf{0 . 4 4}(0.28)$ & $\mathbf{0 . 4 8}(\mathbf{0 . 2 8})$ & $\mathbf{0 . 5 0 9}(\mathbf{0 . 3 5 5})$ \\
\hline Baseline $(\mathrm{s})^{2}(\mathrm{p})(\mathrm{o})^{2}$ & $0.64(0.54)$ & $0.44 \mathbf{( 0 . 3 2 )}$ & $\mathbf{0 . 4 4 ( \mathbf { 0 . 2 9 } )}$ & $0.46(0.26)$ & $0.495(\mathbf{0 . 3 5 5})$ \\
\hline
\end{tabular}

Table 4.nDCG@100 (@10) for different approaches to extend the index.

\begin{tabular}{l|l|l|l|l|l}
\hline Method & SemSearch_ES & INEX-LD & QALD2 & ListSearch & AVG \\
\hline Extended-label $(\mathrm{s})(\mathrm{p})(\mathrm{o})^{2}$ & $0.67(\mathbf{0 . 5 6})$ & $0.45(0.31)$ & $0.44(0.28)$ & $0.48(0.28)$ & $0.510(0.358)$ \\
\hline Extended-comment $(\mathrm{s})(\mathrm{p})(\mathrm{o})^{2}$ & $\mathbf{0 . 6 8}(\mathbf{0 . 5 6})$ & $\mathbf{0 . 5 3}(\mathbf{0 . 3 7})$ & $\mathbf{0 . 5 0}(\mathbf{0 . 3 4})$ & $\mathbf{0 . 5 4}(\mathbf{0 . 3 4})$ & $\mathbf{0 . 5 6 2}(\mathbf{0 . 4 0 3})$ \\
\hline Extended-outgoing $(\mathrm{s})(\mathrm{p})(\mathrm{o})^{2}$ & $0.61(0.52)$ & $0.45(0.34)$ & $0.43(0.32)$ & $0.49(0.33)$ & $0.495(0.378)$ \\
\hline
\end{tabular}

Table 5. nDCG@100 (@10) for different similarity models.

\begin{tabular}{l|l|l|l|l|l}
\hline Module & SemSearch_ES & INEX-LD & QALD2 & ListSearch & AVG \\
\hline BM25 & $0.68(0.56)$ & $0.53(0.37)$ & $\mathbf{0 . 5 0}(\mathbf{0 . 3 4})$ & $0.54(0.34)$ & $0.562(0.403)$ \\
\hline DFR & $\mathbf{0 . 7 2}(\mathbf{0 . 6 1})$ & $\mathbf{0 . 5 5}(0.38)$ & $\mathbf{0 . 5 0}(0.33)$ & $0.53(0.33)$ & $0.575(0.412)$ \\
\hline LM Dirichlet & $0.42(0.38)$ & $0.31(0.26)$ & $0.29(0.23)$ & $0.31(0.23)$ & $0.333(0.275)$ \\
\hline LM Jelinek-Mercer & $0.71(0.59)$ & $\mathbf{0 . 5 5}(\mathbf{0 . 3 9})$ & $\mathbf{0 . 5 0}(\mathbf{0 . 3 4})$ & $\mathbf{0 . 5 5}(\mathbf{0 . 3 5})$ & $\mathbf{0 . 5 7 8}(\mathbf{0 . 4 1 7})$ \\
\hline
\end{tabular}

outgoing properties of the subject and object URIs as two different fields. The object is enriched only if it is a URI. We do not enrich the predicate because the used collection does not include triples that describe the property URIs.

Table 4 shows the results. We see that including the comment property improves performance by more than 5\%. On the contrary, including all outgoing properties drops the performance from 0.510 to 0.495 , which means that this extension method introduces noise. With respect to the label property, we see that performance is almost the same. This is an expected result given that, in the DBpedia collection, for the majority of resources the last part of the URI is similar to the value of the $\mathrm{rdfs}$ : label property.

Examining Different Similarity Models. We now study the effect of the different similarity models offered by Elasticsearch (on their default setting), as described in Sect. 4.4: BM25 $(k 1=1.2, b=0.75)$, DFR (basic model: $g$, after effect: $l$, normalization: $z)$, LM Dirichlet $(\mu=2,000)$, and LM Jelinek-Mercer $(\lambda=0.1)$. Since the performance of a similarity model is highly affected by the indexed data, we consider the best performing extended method of our previous experiments, i.e., extended-comment $(s)(p)(o)^{2}$.

Table 5 shows the results. We notice that three of the models (BM25, DFR, and LM Jelinek-Mercer) have a very similar performance, with LM JelinekMercer outperforming the other two in all query categories apart from SemSearch_ES, the simplest category, for which DFR provides the best results.

Comparative Results on the Full Collection. We now examine the performance of our approach on the full collection and compare it to a set of other available approaches in the bibliography that focus on entity search in DBpedia. 
Specifically, we consider the best performing methods for baseline and extended approaches: baseline $(s)(p)(o)^{2}$ and extended-comment $(s)(p)(o)^{2}$ respectively, with both BM25 and LM Jelinek-Mercer similarity models.

Since the proposed methods do not require training, we compare them with the unsupervised methods of [10] (BM25, PRMS, MLM-all, LM, SDM). Note also that all the methods in [10] have been particularly designed for entity search in DBpedia and, as described in the dataset's github repository ${ }^{7}$, a set of more than 25 DBpedia-specific properties was collected for representing an entity and creating the index. On the contrary, we provide general methods that consider an existing IRS (using triple as the retrieval unit), that do not require special dataset-specific information for building the indexes, apart from the use of a very common property, like rdfs: comment.

Table 6. nDCG@100 (nDCG@10) results on full collection.

\begin{tabular}{|c|c|c|c|c|c|}
\hline Method & SemSearch_ES & INEX-LD & QALD2 & ListSearch & AVG \\
\hline Elas4RDF $_{B L}$ BM25 & $\begin{array}{l}0.67 \\
(0.57)\end{array}$ & $\begin{array}{l}0.45 \\
(0.34)\end{array}$ & $\begin{array}{l}0.32 \\
(0.23)\end{array}$ & $\begin{array}{l}0.37 \\
(0.27)\end{array}$ & $\begin{array}{l}0.455 \\
(0.352)\end{array}$ \\
\hline $\begin{array}{l}\text { Elas4RDF } E X T \\
\text { BM25 }\end{array}$ & $\begin{array}{l}0.68 \\
(0.59)\end{array}$ & $\begin{array}{l}0.48 \\
(0.38)\end{array}$ & $\begin{array}{l}0.41 \\
(0.29)\end{array}$ & $\begin{array}{l}0.43 \\
(0.30)\end{array}$ & $\begin{array}{l}0.500 \\
(0.390)\end{array}$ \\
\hline $\begin{array}{ll}\text { Elas } \mathrm{RDF}_{B L} & \mathrm{LM} \\
\text { Jelinek-Mercer } & \end{array}$ & $\begin{array}{l}0.67 \\
(0.56) \\
\end{array}$ & $\begin{array}{l}0.44 \\
(0.32) \\
\end{array}$ & $\begin{array}{l}0.37 \\
(0.25)\end{array}$ & $\begin{array}{l}0.37 \\
(0.25)\end{array}$ & $\begin{array}{l}0.463 \\
(0.345)\end{array}$ \\
\hline $\begin{array}{l}\text { Elas4RDF }_{E X T} \quad \text { LM } \\
\text { Jelinek-Mercer }\end{array}$ & $\begin{array}{l}0.68 \\
(0.59)\end{array}$ & $\begin{array}{l}0.46 \\
(0.36) \\
\end{array}$ & $\begin{array}{l}0.41 \\
(0.29)\end{array}$ & $\begin{array}{l}0.41 \\
(0.29) \\
\end{array}$ & $\begin{array}{l}0.490 \\
(0.382)\end{array}$ \\
\hline $\begin{array}{l}\text { DBpedia-Entity-v2 } \\
\text { BM25 }\end{array}$ & $\begin{array}{l}0.41 \\
(0.24)\end{array}$ & $\begin{array}{l}0.36 \\
(0.27)\end{array}$ & $\begin{array}{l}0.33 \\
(0.27)\end{array}$ & $\begin{array}{l}0.33 \\
(0.21)\end{array}$ & $\begin{array}{l}0.358 \\
(0.255)\end{array}$ \\
\hline $\begin{array}{l}\text { DBpedia-Entity-v2 } \\
\text { PRMS }\end{array}$ & $\begin{array}{l}0.61 \\
(0.53)\end{array}$ & $\begin{array}{l}0.43 \\
(0.36)\end{array}$ & $\begin{array}{l}0.40 \\
(0.32)\end{array}$ & $\begin{array}{l}0.44 \\
(0.37)\end{array}$ & $\begin{array}{l}0.469 \\
(0.391)\end{array}$ \\
\hline $\begin{array}{l}\text { DBpedia-Entity-v2 } \\
\text { MLM-all }\end{array}$ & $\begin{array}{l}0.62 \\
(0.55)\end{array}$ & $\begin{array}{l}0.45 \\
(0.38)\end{array}$ & $\begin{array}{l}0.42 \\
(0.32)\end{array}$ & $\begin{array}{l}0.46 \\
(0.37)\end{array}$ & $\begin{array}{l}0.485 \\
(0.402)\end{array}$ \\
\hline $\begin{array}{l}\text { DBpedia-Entity-v2 } \\
\text { LM }\end{array}$ & $\begin{array}{l}0.65 \\
(\mathbf{0 . 5 6})\end{array}$ & $\begin{array}{l}0.47 \\
(\mathbf{0 . 4 0})\end{array}$ & $\begin{array}{l}0.43 \\
(0.34)\end{array}$ & $\begin{array}{l}0.47 \\
(0.39)\end{array}$ & $\begin{array}{l}0.504 \\
(0.418)\end{array}$ \\
\hline $\begin{array}{l}\text { DBpedia-Entity-v2 } \\
\text { SDM }\end{array}$ & $\begin{array}{l}\mathbf{0 . 6 7} \\
(0.55)\end{array}$ & $\begin{array}{l}0.49 \\
(0.40)\end{array}$ & $\begin{array}{l}0.43 \\
(0.34)\end{array}$ & $\begin{array}{l}0.49 \\
(0.40)\end{array}$ & $\begin{array}{l}0.514 \\
(0.419)\end{array}$ \\
\hline
\end{tabular}

Table 6 shows the results. We see that, on average, Elas4RDF achieves the highest performance when using the extended index and BM25. Compared to the DBpedia-Entity-v2 methods, we notice that the performance of our approach is very close to the top-performing SDM method (the difference is 0.014 for nDCG@100 and 0.029 for nDCG@10). SDM performs better on average mainly because of its high performance on the ListSearch query type. This is a rather promising result, given that the DBpedia-Entity-v2 methods are tailored to the DBpedia dataset and the task per se (entity search).

\footnotetext{
${ }^{7}$ https://iai-group.github.io/DBpedia-Entity/index_details.html.
} 


\subsection{Space and Efficiency}

We report the space requirements and the average query execution time of our best models for baseline and extended indexes considering the full DBpedia collection (57 GB uncompressed). The number of virtual documents in both cases is $395,569,688$. The size of the baseline index is around $72 \mathrm{~GB}$ and that of the extended (with rdfs: comment) around $160 \mathrm{~GB}$. We see that, as expected, the extended index requires more than 2 times the size of the baseline index. The average query execution time is around $0.7 \mathrm{~s}$ for the baseline method and $1.6 \mathrm{~s}$ for the extended and depends on the query type. We see that extending the index improves performance, however it affects the space requirements.

\subsection{Executive Summary}

The key findings of the aforementioned experiments are: i) all triple components contribute on achieving the highest performance; ii) object keywords seem to be more important than subject keywords, thus giving higher weight to the object fields can improve performance; iii) extending the index with additional (descriptive) information about the triple URIs improves performance; however, including all available information about the URIs (all outgoing properties) can introduce noise and drop performance; iv) the default similarity model of Elasticsearch (BM25) achieves a satisfactory performance; v) a proper configuration of Elasticsearch can provide a performance very close to that of taskand dataset-specific systems built from scratch.

\section{Conclusion}

The objective of this work was to investigate the use of a classic documentcentric IR system, for enabling keyword search over arbitrary RDF datasets. For this study, we decided to use one of the most widely used IR systems, namely Elasticsearch. To this end, we specified the requirements and identified the main rising questions and issues, related to the selection of the retrieval unit and the data to index. We selected triple as our retrieval unit due to its expressiveness and informativeness, and developed a mapping of a ranked list of triples to a ranked list of entities. Then we experimented with a large number of implementation approaches, including different indexing structures, query types, field-weighting methods and similarity models offered by Elasticsearch. We evaluated the performance of the approaches against the DBpedia-Entity v2 test collection. The results show that Elasticsearch can effectively support keyword search over RDF data if configured properly. The most effective configuration, that weights higher the object part of the triple, performs similarly to systems specifically built for retrieving entities over the DBpedia dataset. This approach is demonstrated in the publicly available Elas4RDF demo ${ }^{8}$.

\footnotetext{
$\overline{8}$ https://demos.isl.ics.forth.gr/elas4rdf/.
} 
One direction that is worth investigating, is the provision of good answers for entity-relation queries, i.e., queries that involve entities that are not directly connected in the indexed RDF graph but they are connected through one or more long paths of triples. In that case, different sets of unconnected triples might be retrieved, each one corresponding to an entity appearing in the query. Thus, in future we plan to study how our approach can be extended for providing answers to such type of queries. Another interesting direction for future work is the automatic detection of the query category and the application of different configuration parameters for each case. Finally, we plan to apply and evaluate our approach in domain-specific RDF datasets, e.g., ClaimsKG [21].

\section{References}

1. Blanco, R., Mika, P., Vigna, S.: Effective and efficient entity search in RDF data. In: Aroyo, L., et al. (eds.) ISWC 2011. LNCS, vol. 7031, pp. 83-97. Springer, Heidelberg (2011). https://doi.org/10.1007/978-3-642-25073-6_6

2. Cheng, G., Qu, Y.: Searching linked objects with Falcons: approach, implementation and evaluation. Int. J. Semant. Web Inf. Syst. (IJSWIS) 5(3), 49-70 (2009)

3. Delbru, R., Campinas, S., Tummarello, G.: Searching web data: an entity retrieval and high-performance indexing model. J. Web Semant. 10, 33-58 (2012)

4. Delbru, R., Rakhmawati, N.A., Tummarello, G.: Sindice at SemSearch 2010. In: WWW. Citeseer (2010)

5. Demartini, G., Kärger, P., Papadakis, G., Fankhauser, P.: L3S research center at the SemSearch 2010 evaluation for entity search track. In: Proceedings of the 3rd International Semantic Search Workshop (2010)

6. Dosso, D., Silvello, G.: A scalable virtual document-based keyword search system for RDF datasets. In: Proceedings of the 42nd International ACM SIGIR Conference on Research and Development in Information Retrieval, pp. 965-968 (2019)

7. Elbassuoni, S., Blanco, R.: Keyword search over RDF graphs. In: International Conference on Information and knowledge management, pp. 237-242. ACM (2011)

8. Elbassuoni, S., Ramanath, M., Schenkel, R., Weikum, G.: Searching RDF graphs with SPARQL and keywords. IEEE Data Eng. Bull. 33(1), 16-24 (2010)

9. Franz, T., Schultz, A., Sizov, S., Staab, S.: TripleRank: ranking semantic web data by tensor decomposition. In: Bernstein, A., et al. (eds.) ISWC 2009. LNCS, vol. 5823, pp. 213-228. Springer, Heidelberg (2009). https://doi.org/10.1007/9783-642-04930-9_14

10. Hasibi, F., et al.: DBpedia-Entity V2: a test collection for entity search. In: SIGIR, pp. 1265-1268. ACM (2017)

11. Ilievski, F., Beek, W., van Erp, M., Rietveld, L., Schlobach, S.: LOTUS: adaptive text search for big linked data. In: Sack, H., Blomqvist, E., d'Aquin, M., Ghidini, C., Ponzetto, S.P., Lange, C. (eds.) ESWC 2016. LNCS, vol. 9678, pp. 470-485. Springer, Cham (2016). https://doi.org/10.1007/978-3-319-34129-3_29

12. Ilievski, F., Beek, W., Van Erp, M., Rietveld, L., Schlobach, S.: LOTUS: linked open text unleashed. In: COLD (2015)

13. Järvelin, K., Kekäläinen, J.: Cumulated gain-based evaluation of IR techniques. ACM Trans. Inf. Syst. (TOIS) 20(4), 422-446 (2002)

14. Johnson, T.: Indexing linked bibliographic data with JSON-LD, BibJSON and elasticsearch. Code4lib J. 19, 1-11 (2013) 
15. Lin, X., Zhang, F., Wang, D.: RDF keyword search using multiple indexes. Filomat 32(5), 1861-1873 (2018). https://doi.org/10.2298/FIL1805861L

16. Liu, X., Fang, H.: A study of entity search in semantic search workshop. In: Proceedings of the 3rd International Semantic Search Workshop (2010)

17. Mountantonakis, M., Tzitzikas, Y.: Large-scale semantic integration of linked data: a survey. ACM Comput. Surv. (CSUR) 52(5), 103 (2019)

18. Ouksili, H., Kedad, Z., Lopes, S., Nugier, S.: Using patterns for keyword search in RDF graphs. In: EDBT/ICDT Workshops (2017)

19. Pérez-Agüera, J.R., Arroyo, J., Greenberg, J., Iglesias, J.P., Fresno, V.: Using BM25F for semantic search. In: Proceedings of the 3rd International Semantic Search Workshop, p. 2. ACM (2010)

20. Shahshahani, M.S., Hasibi, F., Zamani, H., Shakery, A.: Towards a unified supervised approach for ranking triples of type-like relations. In: Pasi, G., Piwowarski, B., Azzopardi, L., Hanbury, A. (eds.) ECIR 2018. LNCS, vol. 10772, pp. 707-714. Springer, Cham (2018). https://doi.org/10.1007/978-3-319-76941-7_66

21. Tchechmedjiev, A., et al.: ClaimsKG: a knowledge graph of fact-checked claims. In: Ghidini, C., et al. (eds.) ISWC 2019. LNCS, vol. 11779, pp. 309-324. Springer, Cham (2019). https://doi.org/10.1007/978-3-030-30796-7_20

22. Tran, T., Cimiano, P., Rudolph, S., Studer, R.: Ontology-based interpretation of keywords for semantic search. In: Aberer, K., et al. (eds.) ASWC/ISWC 2007. LNCS, vol. 4825, pp. 523-536. Springer, Heidelberg (2007). https://doi.org/10. 1007/978-3-540-76298-0_38

23. Tran, T., Wang, H., Rudolph, S., Cimiano, P.: Top-k exploration of query candidates for efficient keyword search on graph-shaped (RDF) data. In: 2009 IEEE International Conference on Data Engineering, ICDE 2009, pp. 405-416. IEEE (2009)

24. Tzitzikas, Y., Manolis, N., Papadakos, P.: Faceted exploration of RDF/S datasets: a survey. J. Intell. Inf. Syst. 48(2), 329-364 (2016). https://doi.org/10.1007/s10844016-0413-8 